\title{
Activation of KRAS promotes the mesenchymal features of basal-type breast cancer
}

\author{
Rae-Kwon Kim ${ }^{1,4}$, Yongjoon Suh ${ }^{1,4}$, Ki-Chun Yoo $^{1}$, Yan-Hong Cui ${ }^{1}$, Hyeonmi Kim ${ }^{1}$, Min-Jung Kim ${ }^{2}$, \\ In Gyu Kim ${ }^{3}$ and Su-Jae Lee ${ }^{1}$
}

Basal-type breast cancers are among the most aggressive and deadly breast cancer subtypes, displaying a high metastatic ability associated with mesenchymal features. However, the molecular mechanisms underlying the maintenance of mesenchymal phenotypes of basal-type breast cancer cells remain obscure. Here, we report that KRAS is a critical regulator for the maintenance of mesenchymal features in basal-type breast cancer cells. KRAS is preferentially activated in basal-type breast cancer cells as compared with luminal type. By loss and gain of KRAS, we found that KRAS is necessary and sufficient for the maintenance of mesenchymal phenotypes and metastatic ability through SLUG expression. Taken together, this study demonstrates that KRAS is a critical regulator for the metastatic behavior associated with mesenchymal features of breast cancer cells, implicating a novel therapeutic target for basal-type breast cancer.

Experimental \& Molecular Medicine (2015) 47, e137; doi:10.1038/emm.2014.99; published online 30 January 2015

\section{INTRODUCTION}

Human breast cancer is a heterogeneous disease characterized by their different pathology, molecular profiles and responsiveness to treatment. On the basis of receptor status such as estrogen receptor (ER), progesterone receptor (PR) and HER2 (ERBB2), breast cancers are classified into luminal A (ER and/ or PR receptor positive, HER2 negative), luminal B (ER and/or PR receptor positive, HER2 positive), HER2 positive (ER/PR negative, HER2 positive) and basal-type tumors (triple-negative breast cancer). ${ }^{1}$ Clinical therapies that target the drivers of individual breast cancers have substantially improved the outcome of women with breast cancer, in particular endocrine therapies for luminal-type cancers that express the estrogen receptor and trastuzumab for cancers with HER2 amplification. $^{2}$ In contrast, basal-type breast cancers do not respond to hormonal therapy such as tamoxifen, aromatase inhibitors or therapies that target HER2 receptors such as Herceptin. In addition, basal-type breast cancer is highly proliferative with a short time from relapse to death. ${ }^{3}$ Basaltype breast cancer cells display mesenchymal features that are highly migratory and have invasive properties compared with the luminal type. In line with these observations, expression of epithelial-mesenchymal cell transition (EMT) markers and regulators are closely associated with the basal-type breast cancer cells. ${ }^{4,5}$ However, the molecular mechanisms underlying the maintenance of mesenchymal phenotypes and the metastatic ability of basal-type breast cancer cells remain obscure.

KRAS (Kirsten rat sarcoma viral oncogene homolog) is a small GTPase that transduces extracellular signals to the nucleus, acting as molecular switches in signaling networks that connect a variety of upstream signals to a wide array of downstream signaling pathways associated with cell differentiation, growth, survival, cell-cycle progression, migration and cytoskeletal changes. ${ }^{6-8}$ The balance among this plethora of signaling pathways is crucial in determining the final cellular outcome. Aberrant KRAS signaling has a role in $30 \%$ of all human cancers, with the highest incidence of activating mutations found in pancreatic (70-90\%), colon (30-50\%) and lung (20-30\%) carcinomas. $^{9-11}$ However, in breast cancers, the importance of KRAS relatively has not been intensively focused due to its low frequency of mutation $(<5 \%)$.

In this study, we report that KRAS is a critical regulator for the maintenance of mesenchymal features in basal-type breast cancer cells. By comparison of KRAS activity between

${ }^{1}$ Department of Life Science, Research Institute for Natural Sciences, Hanyang University, Seoul, Korea; ${ }^{2}$ Laboratory of Radiation Exposure \& Therapeutics, National Radiation Emergency Medical Center, Korea Institute of Radiological and Medical Sciences, Seoul, Korea and ${ }^{3}$ Department of Radiation Biology, Environmental Radiation Research Group, Korea Atomic Energy Research Institute, Daejeon, Korea

${ }^{4}$ These authors contributed equally to this work.

Correspondence: Professor S-J Lee, Laboratory of Molecular Biochemistry, Department of Life Science, Hanyang University, 17 Haengdang-Dong, Seongdong-Ku, Seoul 133-791, Korea.

E-mail: sj0420@hanyang.ac.kr

Received 27 August 2014; revised 15 October 2014; accepted 28 October 2014 
basal- and luminal-type of breast cancer cells, we show that the activity of KRAS is preferentially higher in basal-type breast cancer cells compared with the luminal type. We also demonstrate that KRAS is critical for the metastatic ability associated with mesenchymal phenotypes of basal-type breast cancer cells, suggesting that KRAS signaling is a novel therapeutic target for basal-type breast cancer.

\section{MATERIALS AND METHODS}

\section{Cell culture}

Human breast epithelial cell line MCF10A, breast cancer cell lines, MCF-7, SK-BR3, BT549, BT474, T47D, MDA-MB-231, Hs578t and BT20 were established from the American Type Culture Collection (Manassas, VA, USA). Cells were cultured in a humidified $5 \% \mathrm{CO}_{2}$ atmosphere at $37^{\circ} \mathrm{C}$. The normal human breast epithelial cell line MCF10A was maintained in Dulbecco's Modified Eagle's/F-12 medium supplemented with 5\% heat-inactivated horse serum (Invitrogen, Seoul, Korea), $10 \mu \mathrm{g} \mathrm{ml}^{-1}$ insulin, $20 \mathrm{ng} \mathrm{ml}^{-1}$ EGF, $0.1 \mu \mathrm{g} \mathrm{ml}^{-1}$ cholera toxin, $0.5 \mu \mathrm{g} \mathrm{ml}^{-1}$ hydrocortisone, penicillin (100 units per $\mathrm{ml}$ ) and streptomycin $\left(100 \mu \mathrm{g} \mathrm{ml}^{-1}\right)$. MCF7 cells were grown in minimum Eagle's medium supplemented with $10 \%$ fetal bovine serum, penicillin ( 100 units per $\mathrm{ml})$ and streptomycin $\left(100 \mathrm{~g} \mathrm{ml}^{-1}\right)$. MDA-MB-231, BT474, T47D, Hs578t and SK-BR3 cells were grown in Dulbecco's Modified Eagle's medium; BT549 and BT20 cells in RPMI supplemented with $10 \%$ fetal bovine serum, penicillin (100 units per $\mathrm{ml})$ and streptomycin $\left(100 \mathrm{~g} \mathrm{ml}^{-1}\right)$.

\section{Chemical reagents and antibodies}

Polyclonal antibodies to phospho-AKT (Ser-473), phospho-AKT (Thr-308), phospho-ERK1/2 (Thr-202/Tyr-204), ERK and N-cadherin were obtained from Cell Signaling Technology (Beverly, MA, USA). Polyclonal antibodies to AKT, Fibronectin (FN1), TWIST, ZEB1, SNAIL and SLUG were purchased from Santa Cruz Biotechnology (Santa Cruz, CA, USA). The polyclonal antibody Vimentin (VIM) was obtained from Thermo Scientific (Seoul, Korea). 4,6-Diamidino-2phenylindole and monoclonal antibodies to $\beta$-actin were obtained from Sigma (St Louis, MO, USA). Anti-mouse Alexa Fluor 488, and anti-rabbit Alexa Fluor 488 were purchased from Invitrogen. E-cadherin was obtained from BD Biosciences (San Jose, CA, USA). KRAS was purchased from Abcam (Seoul, Korea).

\section{Western blot analysis}

Cell lysates were prepared by extracting proteins with lysis buffer (40 mM Tris- $\mathrm{HCl}$ ( $\mathrm{pH}$ 8.0), $120 \mathrm{~mm} \mathrm{NaCl,} \mathrm{0.1 \%} \mathrm{Nonidet-P40)}$ supplemented with protease inhibitors. Proteins were separated by SDS-polyacrylamide gel electrophoresis, and transferred to a nitrocellulose membrane (Amersham, Arlington Heights, IL, USA). The membrane was blocked with $5 \%$ non-fat dry milk in Tris-buffered saline and incubated with primary antibodies overnight at $4{ }^{\circ} \mathrm{C}$. The blots were developed with a peroxidase-conjugated secondary antibody, and proteins were visualized by enhanced chemiluminescence procedures (Amersham), using the manufacturer's protocol.

\section{Activated KRAS affinity precipitation assay}

Activated RAS affinity precipitation assay was performed according to the manufacturer's protocol. Briefly, cell lysates were incubated with $5 \mu \mathrm{g}$ of RAF-1 RBD agarose beads (upstate, Charlotsville, VA, USA) for $30 \mathrm{~min}$ at $4{ }^{\circ} \mathrm{C}$. After extensive washing of the agarose beads three times with washing buffer (25 mM HEPES ( $\mathrm{pH} 7.5$ ), $10 \mathrm{~mm} \mathrm{MgCl}_{2}$,
$150 \mathrm{~mm} \mathrm{NaCl}, 1 \mathrm{~mm}$ EDTA, 1\% Nonidet P-40, 1 mм Na3VO 4 , 10\% glycerol, $10 \mu \mathrm{g} \mathrm{ml}^{-1}$ leupeptin, $10 \mu \mathrm{g} \mathrm{ml}^{-1}$ aprotinin and $25 \mathrm{~mm} \mathrm{NaF}$ ), the activated KRAS (GTP-RAS) bound to RAF-1 RBD argarose beads was released by the addition of SDS-polyacrylamide gel electrophoresis sample buffer. The amount of activated KRAS was determined by immunoblotting with a KRAS antibody.

\section{Transfection}

Small interference RNA (siRNA) duplexes were introduced into cells using Lipofectamine 2000 reagent (Invitrogen, Carlsbad, CA, USA) according to the procedure recommended by the manufacturer. Cells were collected after $48 \mathrm{~h}$ for subsequent experiments. All siRNA were purchased from Samchully Pharmaceutical Co. Ltd. (Seoul, Korea).

\section{Transduction}

Oncogenic KRAS (G13D) was cloned into retroviral vector MFG. For retrovirus production, H29D cells were cultured in Dulbecco's Modified Eagle's medium (Invitrogen) supplemented with 10\% fetal bovine serum, $2 \mathrm{mmoll}^{-1}$ GlutaMAX (Invitrogen), 50 units per $\mathrm{ml}$ penicillin/streptomycin, $1 \mu \mathrm{g} \mathrm{ml}^{-1}$ tetracycline, $2 \mu \mathrm{g} \mathrm{ml}^{-1}$ puromycin and $0.6 \mathrm{mg} \mathrm{ml}^{-1} \mathrm{G} 418$ sulfate (Calbiochem, San Diego, CA, USA) and transfected with MFG or MFG-KRAS using the Lipofectamine 2000 reagent (Invitrogen). Forty-eight hours after the transfection, viral supernatant was harvested and passed through a $0.45-\mu \mathrm{m}$ filter, and the viral supernatant was frozen at $-80^{\circ} \mathrm{C}$. The supernatant was used for infection after adding $4 \mu \mathrm{g} \mathrm{ml}^{-1}$ Polybrene (Sigma).

\section{Immunocytochemistry}

Cells were fixed with $4 \%$ paraformaldehyde and permeabilized with $0.1 \%$ Triton X-100 in phosphate-buffered saline. Following cell fixation, cells were incubated with the appropriate primary antibodies in a solution of phosphate-buffered saline with $1 \%$ bovine serum albumin and $0.1 \%$ Triton $\mathrm{X}-100$ at $4{ }^{\circ} \mathrm{C}$ overnight. Antibodies used were as follows: human anti-E-cadherin (mouse polyclonal antibody, 1:200), -N-cadherin (mouse polyclonal antibody, 1:200), -VIM (mouse polyclonal antibody, 1:200) and -FN1 (mouse polyclonal antibody, 1:200). Staining was visualized using anti-rabbit or antimouse Alexa Flour 488 (Molecular Probes, Seoul, Korea). Nuclei were counterstained using 4,6-diamidino-2-phenylindole (Sigma). Stained cells were visualized with a fluorescence-microscope (Olympus IX71).

\section{Invasion and migration assays}

Cells $\left(2 \times 10^{4}\right.$ cells per well $)$ were suspended in $0.2 \mathrm{ml}$ of growth medium for invasion and migration assays. For invasion assay, the cells were loaded in the upper well of the transwell chamber $(8-\mathrm{mm}$ pore size; Corning Glass, Seoul, Korea) that was precoated with 10 $\mathrm{mg} \mathrm{ml}^{-1}$ growth factor-reduced matrigel (BD Biosciences) on an upper side of the chamber with the lower well filled with $0.8 \mathrm{ml}$ of growth medium. After incubation for $48 \mathrm{~h}$ at $37^{\circ} \mathrm{C}$, non-invaded cells on the upper surface of the filter were removed with a cotton swab, and migrated cells on the lower surface of the filter were fixed and stained with a Diff-Quick kit (Fisher, Pittsburgh, PA, USA) and photographed (magnification $\times 20$ ). Invasiveness was determined by counting cells in five microscopic fields per well, and the extent of invasion was expressed as an average number of cells per microscopic field. Cells were imaged by phase contrast microscopy (Leica Microsystems, Bannockburn, IL, USA). For migration assay, we used the transwell chambers with inserts that contained the same type of membrane but without the matrigel coating. 


\section{Wound-healing assay}

Cells were plated in $60 \mathrm{~mm}$ culture dish and grown to $80 \%$ confluence in complete medium. A 'wound' was made by scraping with a P200/yellow pipette tip in the middle of the cell monolayer. Floating cells were removed by washing with phosphate-buffered saline and fresh complete medium was added. Cells were incubated at $37^{\circ} \mathrm{C}$ for $24 \mathrm{~h}$. Cells were imaged with by phase-contrast microscopy (Leica Microsystems).

\section{Animal experiments}

MDA-MB231 $\left(1 \times 10^{6}\right.$ cells $)$ cells were injected into the tail vein of athymic BALB/c nude mice $(n=4$ per group; Orient, Gyeonggido, Korea). Lung metastasis was analyzed by counting the number of foci on the lung surface with naked eyes. This study was reviewed and approved by the Institutional Animal Care and Use Committee (IACUC) of Center for Laboratory Animal Sciences, Medical Research Coordinating Center, HYU Industry-University Cooperation Foundation.

\section{Statistical analysis}

All experimental data are reported as mean and the error bars represent the experimental standard error. Statistical analysis was performed by the nonparametric Student $t$-test.

\section{RESULTS}

KRAS is a critical regulator for invasiveness of basal-type breast cancer cells

To examine whether KRAS is correlated with basal-type breast cancer cells, we compared the activation status and expression levels of KRAS between luminal-type and basal-type breast cancer cell lines. Since the GTP-bound, active form of KRAS can interact with RAF-1, KRAS activation was assessed by quantifying the amount of KRAS that interacts with RAF-1. Importantly, malignant basal-type (MDA-MB231, BT549, Hs578t and BT20) breast cancer cells had a higher activity as well as higher expression levels of KRAS compared with luminal-type breast cancer cell lines (BT474, MCF7, SKBR3 and T47D; Figure 1a). Among these breast cancer cell lines, only MDA-MB231 cells carry KRAS mutation; KRAS G13D. ${ }^{12}$ Since basal-type breast cancer cells display more invasive and migratory properties, we next examined whether higher activation of KRAS contributes to these malignant phenotypes in basal-type breast cancer cells. To this end, we chose two basal-type breast cancer cell lines, MDA-MB231 and BT549. Although BT549 cells carry wild-type KRAS, the cells displayed high activity of KRAS to the levels comparable with that of MDA-MB231 cells harboring KRAS mutant form. Importantly,

a

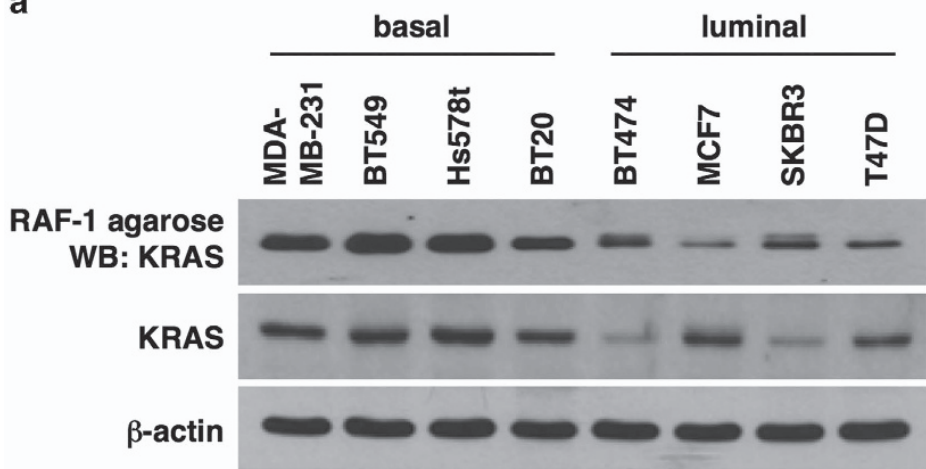

b

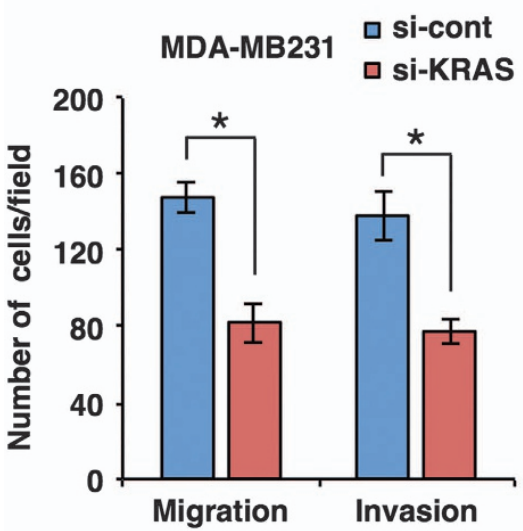

C
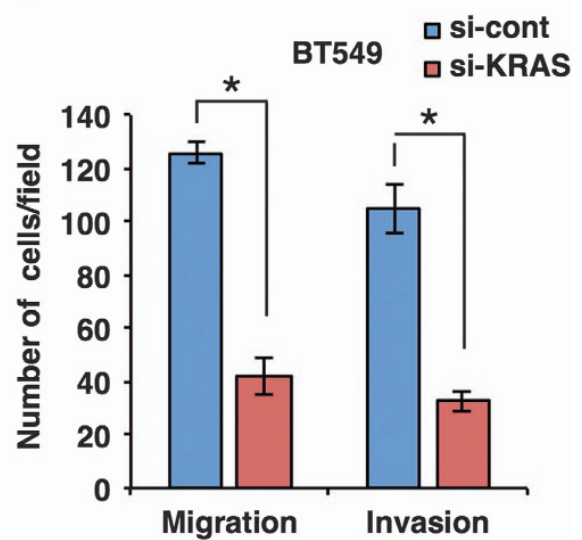

Figure 1 KRAS activity is critical for mesenchymal phenotypes of basal-type breast cancer cells. (a) Activated KRAS affinity precipitation assay and western blot for KRAS comparing basal- and luminal-type of breast cancer cell lines. Migration and invasion assay of MDAMB231 (b) and BT549 (c) basal-type breast cancer cells after treatment with siRNA targeting KRAS. $\beta$-Actin was used as a loading control. Error bars represent mean \pm s.d. of triplicate samples. KRAS, Kirsten rat sarcoma viral oncogene homolog; si-cont, scrambled control siRNA; si-KRAS, siRNA targeting KRAS. ${ }^{*} P<0.01$ versus control. 
siRNA-mediated downregulation of KRAS effectively attenuated migratory and invasive properties both in MDA-MB231 and BT549 cells (Figures $1 \mathrm{~b}$ and c). Taken together, these results suggest that KRAS is preferentially expressed and activated in basal-type breast cancer cells and contributes to invasiveness of basal-type breast cancer cells.

KRAS is necessary for the maintenance of mesenchymal phenotypes of basal-type breast cancer cells

Tumor cell invasion is involved with the loss of cell-cell interaction together with the acquisition of migratory properties, and is often associated with EMT. ${ }^{13}$ Very recently, several lines of evidence suggested that expression of EMT markers and regulators are closely associated with the basal-type breast cancer cells. ${ }^{4,5}$ We next examined whether KRAS promotes invasiveness of basal-type breast cancer cells through EMT. Notably, siRNA-mediated downregulation of KRAS caused a decrease in the mesenchymal cell markers such as VIM and FN1 in MDA-MB231 cells (Figures 2a and b). In the BT549 cell line, downregulation of KRAS caused an increase in the epithelial cell marker E-cadherin and a decrease in the mesenchymal cell markers N-cadherin and VIM. We next examined EMT transcription factors, such as SLUG, SNAIL, ZEB1 and TWIST, after treatment with siRNA targeting KRAS in basal-type breast cancer cells. Notably, SLUG expression was markedly decreased by downregulation of KRAS, whereas SNAIL, ZEB1 and TWIST expression were not altered, indicating that KRAS promotes EMT through SLUG in breast cancer cells (Figure 2c). Taken together, these results suggest that activation status of KRAS is critical for the mesenchymal phenotype and metastatic ability of basal-type breast cancer cells.

\section{Ectopic expression of KRAS confers mesenchymal} phenotypes on luminal-type breast cancer cells

Since KRAS is necessary for the maintenance of mesenchymal phenotypes in basal-type breast cancer cells, we next examined whether ectopic expression of KRAS confers mesenchymal phenotypes on luminal-type breast cancer cells. To this end, we overexpressed oncogenic mutant form of KRAS G13D in relatively less malignant luminal-type MCF7 breast cancer cells (Figure 3a). We next examined whether exogenous KRAS transduces the downstream signaling pathways of KRAS. Importantly, retrovirus-mediated delivery of the oncogenic KRAS enhanced the kinase activity of RAF-1 and increased the phosphorylation of ERK (Figure 3b). Also, oncogenic KRAS expression increased the activity of PI3K and phosphorylation of AKT on Thr308 and Ser473 (Figure 3c). Thus, a

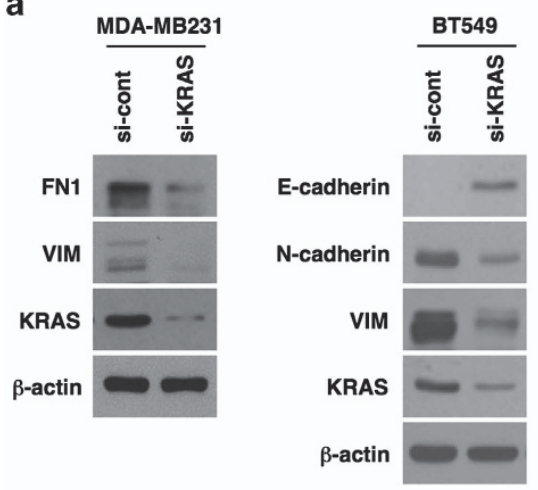

C

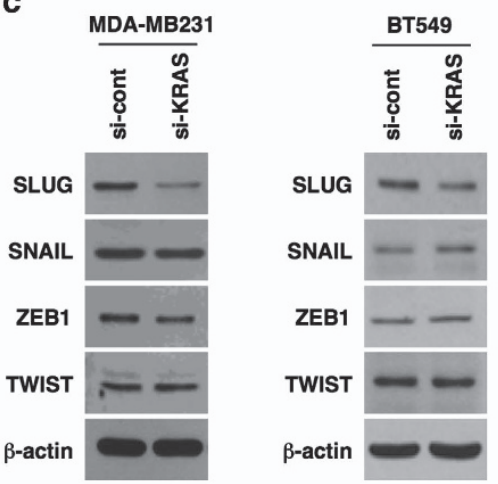

b

MDA-MB231
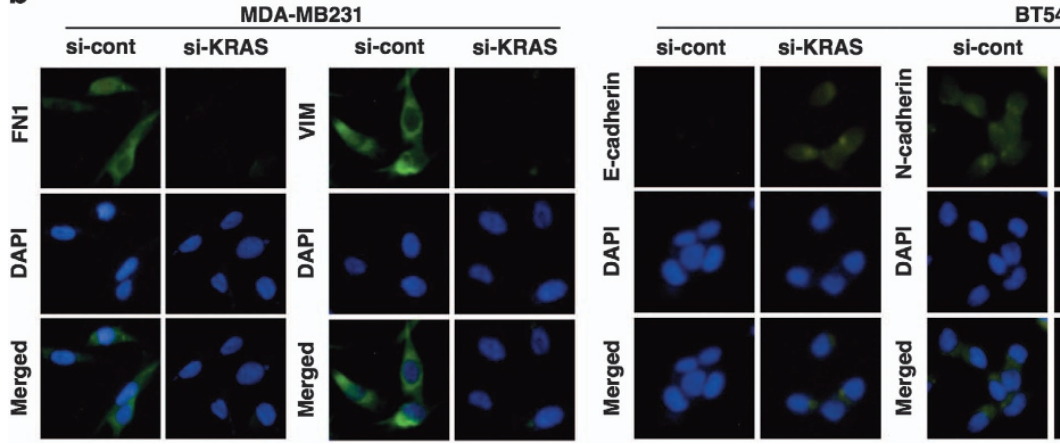

549
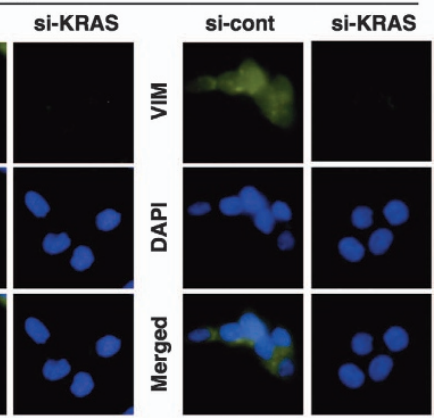

Figure 2 KRAS is necessary for the maintenance of mesenchymal features of basal-type breast cancer cells. Western blot analysis (a) and immunocytochemistry (b) for EMT markers such as FN1, VIM, E-cadherin and N-cadherin in basal-type breast cancer cells after treatment with siRNA targeting KRAS. (c) Western blot analysis for EMT master regulators such as SLUG, SNAIL, ZEB1 and TWIST in basal-type MDA-MB231 breast cancer cells after treatment with siRNA targeting KRAS. $\beta$-Actin was used as a loading control. DAPI, 4, 6-diamidino2-phenylindole; KRAS, Kirsten rat sarcoma viral oncogene homolog; si-cont, scrambled control siRNA; si-KRAS, siRNA targeting KRAS; VIM, vimentin. Error bars represent mean \pm s.d. of triplicate samples. ${ }^{*} P<0.01$ versus control. 


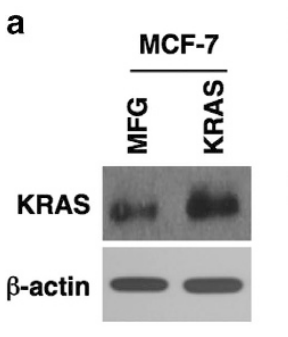

b

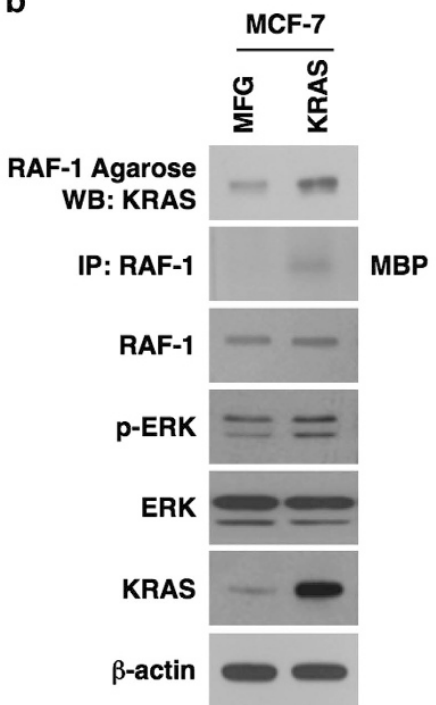

C

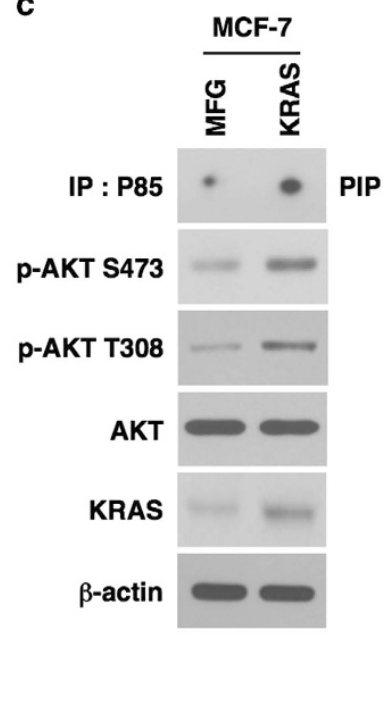

Figure 3 Exogenous expression of KRAS in luminal-type breast cancer cells. Western blot analysis for KRAS (a) and its major downstream effectors, RAF-1/ERK (b) and PI3K/AKT (c) in luminal-type MCF7 breast cancer cells that are transduced with KRAS or control vector MFG. $\beta$-Actin was used as a loading control. KRAS, Kirsten rat sarcoma viral oncogene homolog.

retrovirus-mediated delivery of the oncogenic KRAS caused activation of RAF-1/ERK and PI3K/AKT signaling pathways, the major downstream effectors of KRAS. By overexpression of KRAS, we further found that oncogenic KRAS signaling confers migratory and invasive properties onto MCF7 and SKBR3 luminal-type breast cancer cells (Figures $4 \mathrm{a}$ and b). In parallel, exogenous expression of KRAS caused induction of mesenchymal markers such as N-cadherin and VIM, while it decreased epithelial marker E-cadherin in luminal-type MCF7 breast cancer cells (Figure 4c). Consistent with this data, expression of KRAS in another luminal-type SKBR3 also induced mesenchymal markers such as N-cadherin and VIM. Moreover, KRAS expression induced EMT transcription factor SLUG in both MCF7 and SKBR3 cells (Figure 4d). Taken together, these results suggest that expression of KRAS alone can cause mesenchymal features on luminal-type breast cancer cells.

\section{Oncogenic KRAS promotes mesenchymal phenotypes in normal mammary cells}

To extend our observations, we also transduced normal mammary MCF10A cells with the oncogenic mutant form of KRAS G13D. In line with the above data, expression of oncogenic KRAS G13D also increased the migratory and invasive properties in normal mammary MCF10A cells (Figure 5a), accompanying with EMT markers such as E-cadherin, N-cadherin and VIM (Figure 5b). Notably, as in luminal-type breast cancer cells, overexpression of oncogenic KRAS in MCF10A cells also caused an increase of SLUG, while other EMT transcription factors (SNAIL, ZEB1, TWIST) were not altered by KRAS expression (Figure $5 \mathrm{c}$ ), indicating that KRAS promotes EMT through upregulation of SLUG. Taken together, these results suggest that KRAS also can induce mesenchymal phenotypes in normal mammary cells similar to breast cancer cells.

Knockdown of KRAS suppresses in vivo metastatic ability of basal-type breast cancer cells

As the above data showed that KRAS is a critical regulator for mesenchymal phenotypes and metastatic ability of basal-type breast cancer cells, we next evaluated our findings in vivo. To this end, MDA-MB231 cells were transfected with siRNAtargeting KRAS or scrambled control siRNA, and then injected into the tail veins of athymic nude mice (Figure 6a). By 5 weeks, the frequency of metastasis was analyzed by counting the number of metastatic foci on the surface of whole lungs with naked eyes. Of note, lung metastasis was effectively blocked by the downregulation of KRAS in MDA-MB231 breast cancer cells (Figure 6b). Since metastasis is the primary cause of death in breast cancer patients, we also examined the correlation of KRAS expression levels with prognosis of breast cancer patients. Using an online survival analysis tool, we analyzed the correlation of KRAS expression levels with relapse-free survival rate by the Kaplan-Meier plot. ${ }^{14}$ Importantly, breast cancer patients with higher levels of KRAS displayed significantly a lower relapse-free survival rate compared with patients having relatively lower levels of KRAS (Figure 6c). Collectively, these results suggest that KRAS is a critical regulator for the metastasis of breast cancer cells, having a correlation with prognosis of breast cancer patients.

\section{DISCUSSION}

Basal-type breast cancers are known to be the most aggressive and deadly breast cancer subtypes with high rates of tumor recurrence and poor overall survival., ${ }^{45,16}$ Previous studies have shown that expression of EMT markers and regulators are 
a

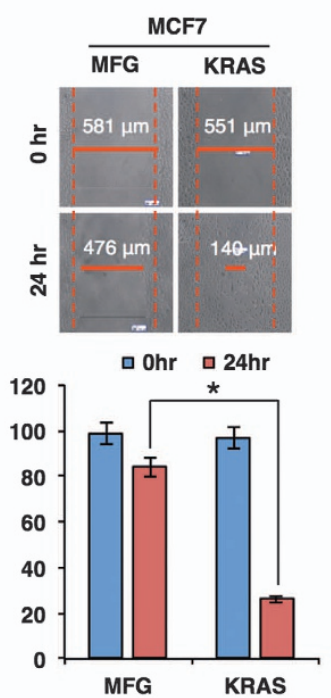

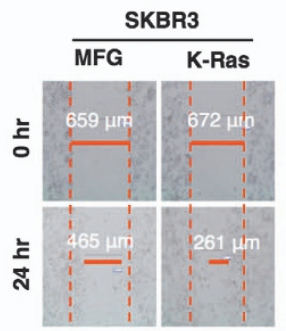

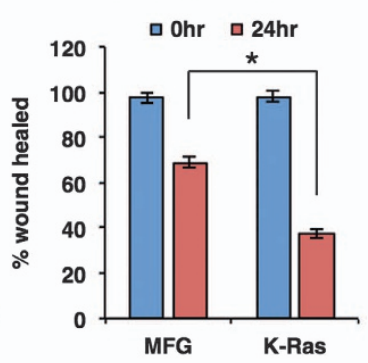

b

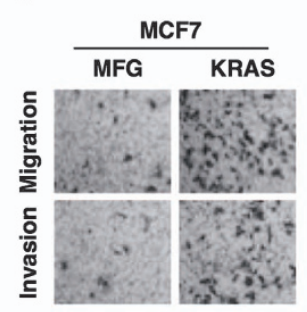

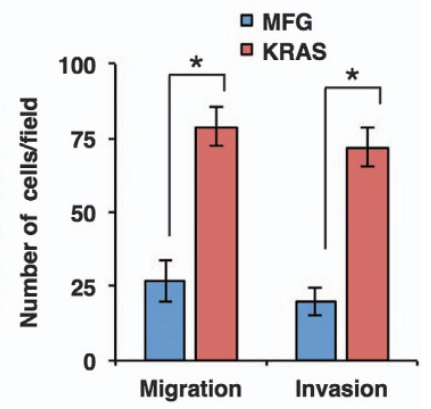
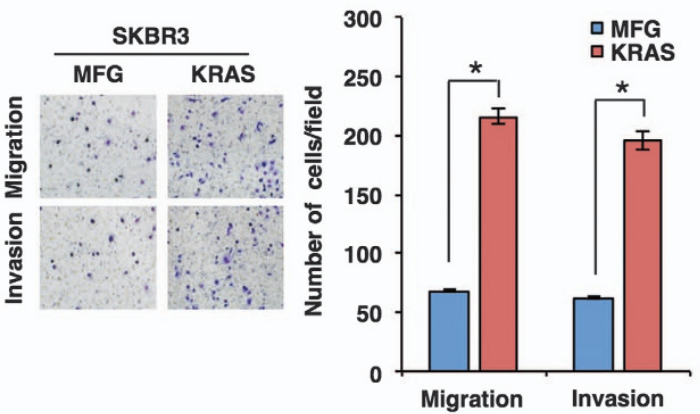

d

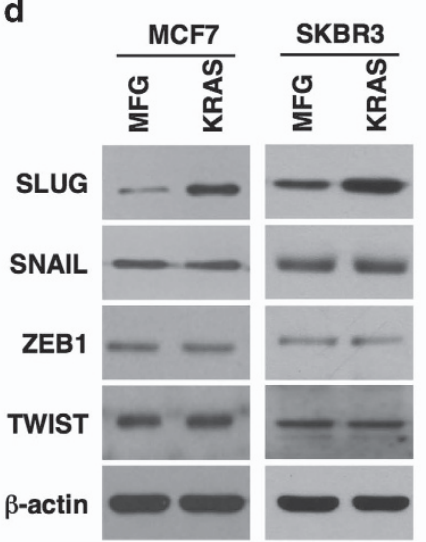

Figure 4 Exogenous expression of KRAS promotes migration and invasion of luminal-type breast cancer cells through EMT. Wound healing (a), migration and invasion assays (b) of MCF7 and SKBR3 luminal-type breast cancer cells that are transduced with KRAS or control vector MFG. Western blot analysis for EMT markers (c) and EMT regulators (d) in KRAS or control vector MFG-transduced MCF7 and SKBR3 luminal-type breast cancer cells. $\beta$-Actin was used as a loading control. Error bars represent mean \pm s.d. of triplicate samples. ${ }^{*} P<0.01$ versus control. KRAS, Kirsten rat sarcoma viral oncogene homolog; VIM, vimentin.

closely associated with the basal-subtype breast cancer cells, indicating that basal-type breast cancer cells display more mesenchymal phenotypes compared with the luminal cancer cells. $^{4,5}$ Because of their mesenchymal features, basal-type breast cancer cells appear to be highly invasive and display metastatic ability. However, the molecular mechanisms underlying the maintenance of mesenchymal phenotypes along with metastatic ability in basal-type breast cancer cells remain obscure.

In this current study, we observed that KRAS signaling has a correlation with characteristics of basal-type breast cancer cells that are more malignant and metastatic subtype. Importantly, both the expression and activity of KRAS were markedly higher in basal-type breast cancer cells than the luminal type, although all of those cancer cells carried wild-type KRAS except MDAMB231 cells. Previously, RAS mutations are found in at least $20 \%$ of all human malignancies, with KRAS being the most frequently activated oncogene. ${ }^{9,10}$ However, despite the observations that KRAS is often aberrantly activated in breast cancers, ${ }^{17}$ the importance of KRAS relatively has not been intensively focused in breast cancers due to its low frequency of mutation. ${ }^{18}$ In this study, by using an endogenous or exogenous KRAS-driven cancer-progression model, we found that aberrant KRAS activation is critical for mesenchymal phenotypes and metastatic ability of basal-type breast cancer cells. Our in vitro and in vivo studies demonstrated that the downregulation of KRAS effectively suppresses mesenchymal phenotypes and metastatic ability of basal-type breast cancer cells. 
a

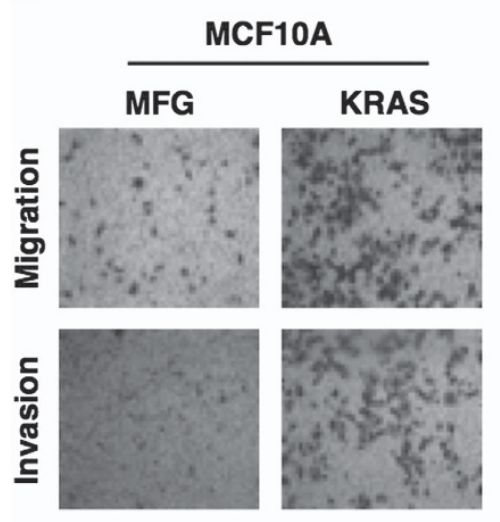

b

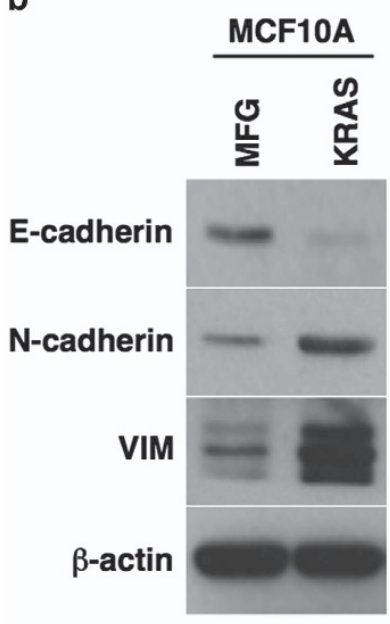

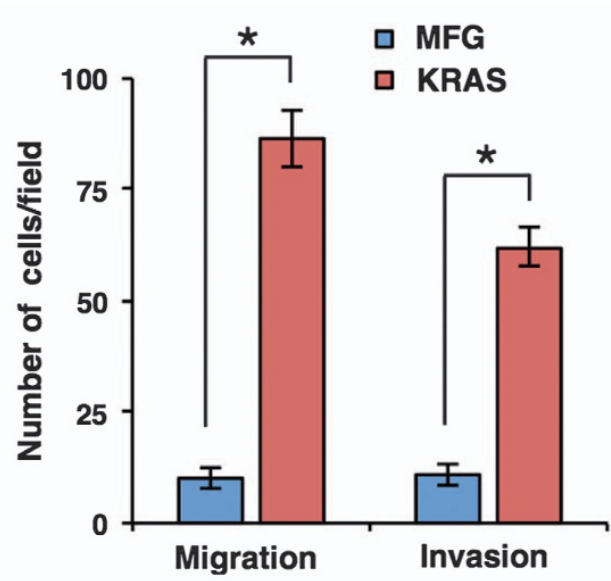

C

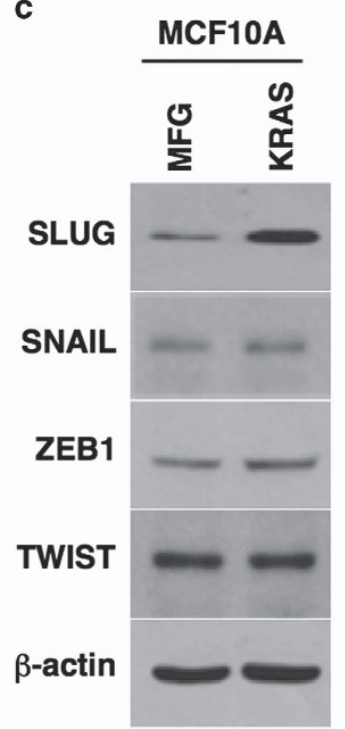

Figure 5 Exogenous expression of KRAS promotes migration and invasion of normal mammary cells through EMT. (a) Migration and invasion assays of MCF1OA normal mammary cells that are transduced with KRAS or control vector MFG. Western blot analysis for EMT markers (b) and EMT regulators (c) in KRAS or control vector MFG-transduced MCF10A normal mammary cells. $\beta$-Actin was used as a loading control. Error bars represent mean \pm s.d. of triplicate samples. ${ }^{*} P<0.01$ versus control. KRAS, Kirsten rat sarcoma viral oncogene homolog; VIM, vimentin.

In contrast, ectopic expression of KRAS conferred mesenchymal phenotypes on luminal-type breast cancer cells as well as normal mammary epithelial cells, suggesting that KRAS signaling is necessary and sufficient for the acquisition of metastatic behavior associated with mesenchymal phenotypes. Supporting our findings, Eckert et al. ${ }^{17}$ found that RAS is frequently activated in breast carcinoma cells that lack mutated RAS. In line with this observation, a functional germline variant in the $3^{\prime}$-UTR of the KRAS oncogene (rs61764370 $\mathrm{T}>\mathrm{G}$ ) has been recently identified and was reported to be associated with increased risk of both invasive epithelial ovarian cancer $^{19}$ and breast cancer ${ }^{20}$ in clinically well-annotated cohorts. This functional variant has been shown to disrupt a let-7 miRNA binding site leading to increased expression of KRAS in vitro. Notably, the frequency of KRAS-variant in breast cancer was found to be associated with basal-type breast cancer. $^{20,21}$ In cancer progression, cancer cells are clonally selected with adopting the most powerful signaling pathways for survival and proliferation. In this regard, the fact that basaltype breast cancer cells have higher levels of KRAS, albeit lower mutation frequency, implicates that KRAS is a strong oncogenic signal that might be adopted by basal-type breast cancer cells, conferring mesenchymal phenotypes on breast cancer cells.

In this current study, we found that downregulation of KRAS decreases mesenchymal features of basal-type breast cancer cells via inhibition of SLUG among EMT transcription factors. Consistently, exogenous expression of KRAS caused an increase of SLUG expression, but not SNAIL, TWIST and ZEB1, in luminal-type breast cancer cells. In agreement with our finding, previous studies suggested that SLUG is highly expressed in basal-type breast cancer cells and is critical to determine basal- and luminal-type breast cancer. ${ }^{22-24}$ Taken together with the previous studies, our findings suggest that 
a

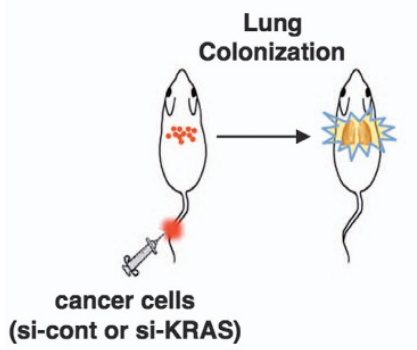

b

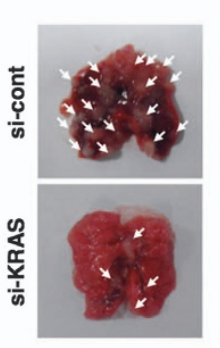

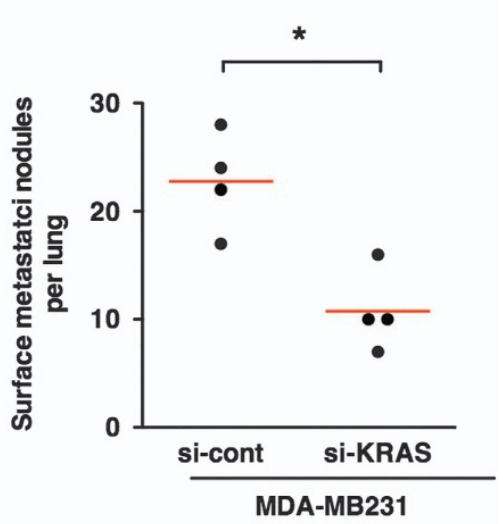

C

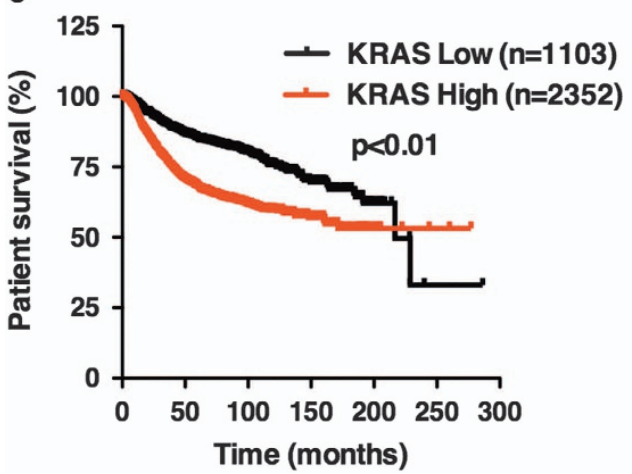

Figure 6 KRAS has a correlation with in vivo metastatic ability of basal-type breast cancer cells and prognosis of breast cancer patients. (a) Schematic experimental procedure for tail vein injection of breast cancer cells that are transfected with siRNA targeting KRAS or scrambled control siRNA into athymic BALB/c nude mice. (b) Representative images of whole-lung metastasis (left) and quantification of the metastases foci (right) generated by MDA-MB231 cells after tail vein injection $(n=4)$. (c) Kaplan-Meier survival curves of human breast cancer patients using publicly available clinical breast cancer database. Breast cancer patients (3455) were grouped to high (2352) and low expression (1103) of KRAS. Note that KRAS expression level has a correlation with poor survival rate. KRAS, Kirsten rat sarcoma viral oncogene homolog. si-cont, scrambled control siRNA; si-KRAS, siRNA targeting KRAS. ${ }^{*} P 0.01$ versus control.

KRAS causes basal-type breast cancer cells to maintain mesenchymal phenotypes through the regulation of SLUG expression.

In summary, KRAS is preferentially activated in basal-type breast cancer cells, compared with the luminal type. By the loss and gain of KRAS, we demonstrated that KRAS is a critical regulator for metastatic behavior associated with mesenchymal features of basal-type breast cancer cells. Importantly, this study showed that KRAS is necessary and sufficient for the maintenance of mesenchymal phenotypes through SLUG expression. Further studies on the KRAS-mediated regulatory mechanism of SLUG might provide a mechanistic insight on the metastatic behavior and therapeutic targets for the malignant subtype of breast cancer cells.

\section{CONFLICT OF INTEREST}

The authors declare no conflict of interest.

\section{ACKNOWLEDGEMENTS}

This work was supported by the National Research Foundation (NRF) and Ministry of Science, ICT \& Future Planning, Korean government, through its National Nuclear Technology Program (NRF-2013M2A2A7066345; NRF-2012M2B2B1055639).

1 Sorlie T, Perou CM, Tibshirani R, Aas T, Geisler S, Johnsen H et al. Gene expression patterns of breast carcinomas distinguish tumor subclasses with clinical implications. Proc Natl Acad Sci USA 2001; 98: 10869-10874.

2 Turner NC, Jones AL. Management of breast cancer-Part II. BMJ 2008; 337: a540.

3 Kennecke H, Yerushalmi R, Woods R, Cheang MC, Voduc D, Speers $\mathrm{CH}$ et al. Metastatic behavior of breast cancer subtypes. J Clin Oncol 2010; 28: 3271-3277.

4 Sarrio D, Rodriguez-Pinilla SM, Hardisson D, Cano A, Moreno-Bueno G, Palacios J. Epithelial-mesenchymal transition in breast cancer relates to the basal-like phenotype. Cancer Res 2008; 68: 989-997.

5 Choi Y, Lee HJ, Jang MH, Gwak JM, Lee KS, Kim EJ et al. Epithelialmesenchymal transition increases during the progression of in situ to invasive basal-like breast cancer. Hum Pathol 2013; 44: 2581-2589.

6 Zuber J, Tchernitsa OI, Hinzmann B, Schmitz AC, Grips M, Hellriegel M et al. A genome-wide survey of RAS transformation targets. Nat Genet 2000; 24: 144-152.

7 Crespo P, Leon J. Ras proteins in the control of the cell cycle and cell differentiation. Cell Mol Life Sci 2000; 57: 1613-1636.

8 Wu Y, Zhuang Y, Han M, Xu T, Deng K. Ras promotes cell survival by antagonizing both JNK and Hid signals in the Drosophila eye. BMC Dev Biol 2009; 9: 53. 
9 Malumbres M, Barbacid M. RAS oncogenes: the first 30 years. Nat Rev Cancer 2003; 3: 459-465.

10 Kranenburg O. The KRAS oncogene: past, present, and future. Biochim Biophys Acta 2005; 1756: 81-82.

11 Yen LC, Uen YH, Wu DC, Lu CY, Yu FJ, Wu IC et al. Activating KRAS mutations and overexpression of epidermal growth factor receptor as independent predictors in metastatic colorectal cancer patients treated with cetuximab. Ann Surg 2010; 251: 254-260.

12 Hollestelle A, Elstrodt F, Nagel JH, Kallemeijn WW, Schutte M. Phosphatidylinositol-3-OH kinase or RAS pathway mutations in human breast cancer cell lines. Mol Cancer Res 2007; 5: 195-201.

13 Thiery JP. Epithelial-mesenchymal transitions in tumour progression. Nat Rev Cancer 2002; 2: 442-454.

14 Gyorffy B, Lanczky A, Eklund AC, Denkert C, Budczies J, Li Q et al. An online survival analysis tool to rapidly assess the effect of 22,277 genes on breast cancer prognosis using microarray data of 1,809 patients. Breast Cancer Res Treat 2010; 123: 725-731.

15 Hudis CA, Gianni L. Triple-negative breast cancer: an unmet medical need. Oncologist 2011; 16(Suppl 1): 1-11.

16 Badve S, Dabbs DJ, Schnitt SJ, Baehner FL, Decker T, Eusebi V et al. Basal-like and triple-negative breast cancers: a critical review with an emphasis on the implications for pathologists and oncologists. Mod Pathol 2011; 24: 157-167.

17 Eckert LB, Repasky GA, Ulku AS, McFall A, Zhou H, Sartor Cl et al. Involvement of Ras activation in human breast cancer cell signaling, invasion, and anoikis. Cancer Res 2004; 64: 4585-4592.

18 Karnoub AE, Weinberg RA. Ras oncogenes: split personalities. Nat Rev Mol Cell Biol 2008; 9: 517-531.

19 Ratner E, Lu L, Boeke M, Barnett R, Nallur S, Chin LJ et al. A KRAS-variant in ovarian cancer acts as a genetic marker of cancer risk. Cancer Res 2010; 70: 6509-6515.
20 Paranjape T, Heneghan H, Lindner R, Keane FK, Hoffman A, Hollestelle A et al. A 3'-untranslated region KRAS variant and triple-negative breast cancer: a case-control and genetic analysis. Lancet Oncol 2011; 12: 377-386.

21 Pilarski R, Patel DA, Weitzel J, McVeigh T, Dorairaj JJ, Heneghan HM et al. The KRAS-variant is associated with risk of developing double primary breast and ovarian cancer. PLoS ONE 2012; 7: e37891.

22 Storci G, Sansone P, Trere D, Tavolari S, Taffurelli M, Ceccarelli C et al. The basal-like breast carcinoma phenotype is regulated by SLUG gene expression. J Pathol 2008; 214: 25-37.

23 Bailey CK, Mittal MK, Misra S, Chaudhuri G. High motility of triple-negative breast cancer cells is due to repression of plakoglobin gene by metastasis modulator protein SLUG. J Biol Chem 2012; 287: 19472-19486.

24 Phillips S, Prat A, Sedic M, Proia T, Wronski A, Mazumdar S et al. Cell-state transitions regulated by SLUG are critical for tissue regeneration and tumor initiation. Stem Cell Reports 2014; 2: 633-647.

(c) $(1) \circledast$ This work is licensed under a Creative Commons Attribution-NonCommercial-NoDerivs 3.0 Unported License. The images or other third party material in this article are included in the article's Creative Commons license, unless indicated otherwise in the credit line; if the material is not included under the Creative Commons license, users will need to obtain permission from the license holder to reproduce the material. To view a copy of this license, visit http://creativecommons.org/licenses/by-nc-nd/3.0/ 